\title{
20 Soft robotics with Variable Stiffness Actuators: Tough robots for soft human robot interaction
}

\author{
Sebastian Wolf, Thomas Bahls, Maxime Chalon, Werner Friedl, Markus \\ Grebenstein, Hannes Höppner, Markus Kühne*, Dominic Lakatos, Nico \\ Mansfeld, Mehmet Can Özparpucu, Florian Petit, Jens Reinecke, Roman \\ Weitschat and Alin Albu-Schäffer
}

German Aerospace Center (DLR)

\begin{abstract}
Robots that are not only robust, dynamic, and gentle in the human robot interaction, but are also able to perform precise and repeatable movements, need accurate dynamics modeling and a high-performance closed-loop control. As a technological basis we propose robots with intrinsically compliant joints, a stiff link structure, and a soft shell. The flexible joints are driven by Variable Stiffness Actuators (VSA) with a mechanical spring coupling between the motor and the actuator output and the ability to change the mechanical stiffness of the spring coupling. Several model based and model free control approaches have been developed for this technology, e.g. Cartesian stiffness control, optimal control, reactions, reflexes, and cyclic motion control.
\end{abstract}

\subsection{Introduction}

Robots interacting with humans in direct physical contact or even acting in place of a human in given situations are of high interest for current research. Manipulating objects in direct contact with the human or in unstructured environment likely results in contacts and collisions that are unpredictable. Furthermore, the desired robot skills include fine manipulation as well as highly dynamic and powerful movements. This results in special demands on the robots capabilities:

- Robustness to fast impacts to reduce the risk of robot damage

- Precision for fine manipulation

- Sensitivity for gentle interaction with the environment

- High dynamics for fast and controlled movements

If the intended application demands the combination of the four aspects, namely to be robust, precise, sensitive, and dynamic at the same time, robots with stiff structures and stiff drive-trains come to their limits. Robots with stiff structure, but inherent compliance in the drive-train, promise to overcome the restrictions of classical stiff robots, being able to combine the four required aspects. The technology of compliant actuators has been intensively investigated in the last decade, which 
gained a great variety of implementations [1]. It can be distinguished between a Variable Impedance Actuator (VIA) with physical variable stiffness and damping, a Variable Stiffness Actuator (VSA) with physical variable stiffness, and a Variable Damping Actuator (VDA) with physical variable damping only.

This article will first introduce the concept of compliant actuation on the example of the DLR Hand Arm System [2]. Then we discuss strategies for control of the hand, Cartesian stiffness control, and optimal control. Furthermore, we show reactions and reflexes to protect the hardware and the human, and address cyclic motion control.

\subsection{Compliant Actuation}

The concept of soft robotics at DLR is based on stiff link structures and compliant elements in the actuator drive-train (see one-dimensional model in Fig. 20.1). A mechanism with a mechanical spring decouples the motor inertia from the output and the link mass, which makes the actuator more robust and enables energy storage in the spring (see Sec. 20.8, [3], [4], [5]). The difference between motor position $\theta$ and link position $q$ is the passive spring deflection $\phi$. Joint torques are calculated by position measurement of the passive spring deflection. The joint torque information is used in control, e.g. impedance control, where the control adds active impedance to the passive compliance of the spring mechanism (see Sec. 20.6.2). There is no passive damping element in the current mechanics, so the actuator types are VSAs. Damping is realized by active control [6], [7]. Most of the link structure is equipped with flexible and padded covers, which give the robot a soft tactile touch and reduce local contact forces.

The anthropomorphic DLR Hand Arm System was built as an experimental platform to evaluate the VSA concept on 3 different types of VSAs [2]. It has a total of 26 DoF with 7 in the arm and 19 in the hand (Fig. 20.2). The flexibility is solely located in the actuators, which are connected with stiff aluminum link structures. The housings are padded with soft foam and textile.

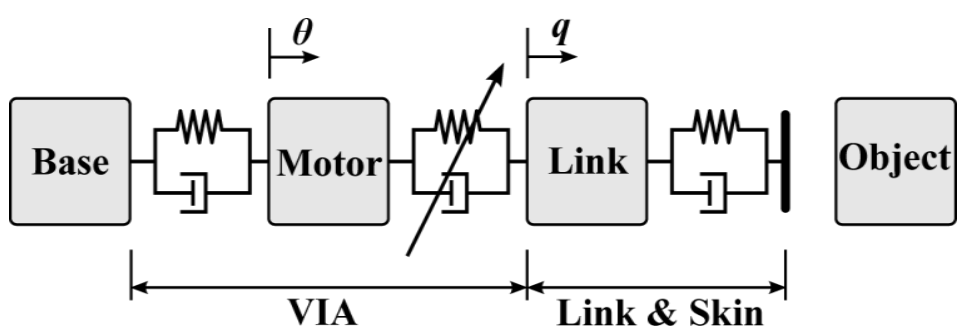

Fig. 20.1 1-DoF mass model of a Variable Impedance Actuator (VIA), which has physical variable stiffness and damping, interacting with an external object. 


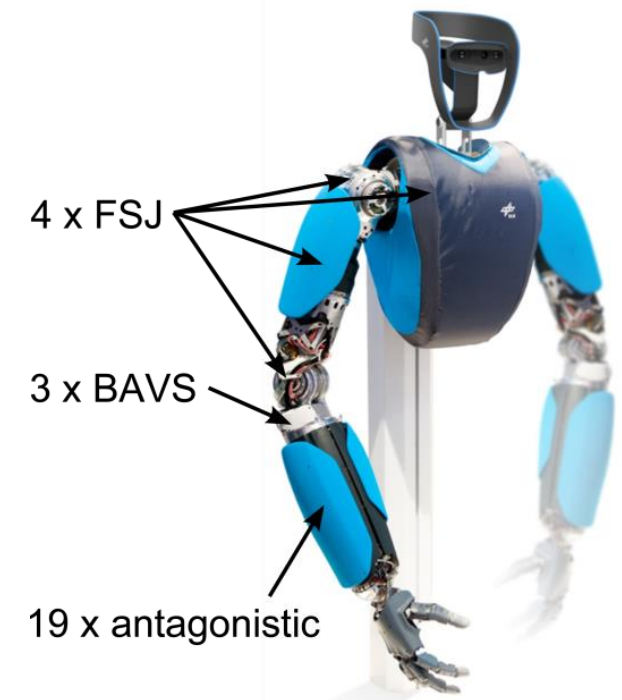

Fig. 20.2 The DLR Hand Arm System is equipped with 3 different types of Variable Stiffness Actuators (VSA)

\subsubsection{Floating Spring Joint (FSJ)}

The mechanical principle of the DLR FSJ (see Fig. 20.3) is a VSA module designed for the first 4 axes of the DLR Hand Arm System [4]. For this purpose, the joints have to be extremely compact to fit into the arm. At the same time they require a high power density in order to approximate the human arm skills. The DLR FSJ is designed to have energy efficient components and low friction. The potential energy of the spring is used to a maximal extend in order to have a high energy capacity to weight ratio. 


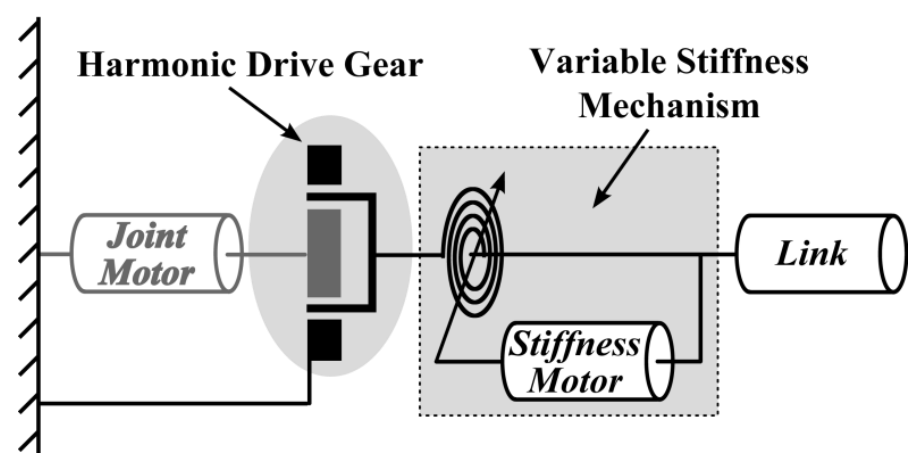

Fig. 20.3 Schematic of the DLR FSJ mechanism. [4]

The DLR FSJ has one big motor to change the output position of the actuator and one small motor to change the stiffness preset of the spring mechanism. In this setup only one motor has to move to change the link position of the robot. If the small motor is kept in a fixed position, the actuator behaves like a Serial Elastic Actuator (SEA). The nonlinear spring mechanism is in series between the main gear and the output.

The torque is generated by a rotational cam disk and roller system which transmits the rotational joint deflection to an axial compression of a linear spring. The shape of the cam disks can be chosen according to the desired torque vs. displacement behavior (see Fig. 20.4). For the DLR Hand Arm System the cam disk shapes were chosen to have a good capability of stiffness variation under all load conditions.

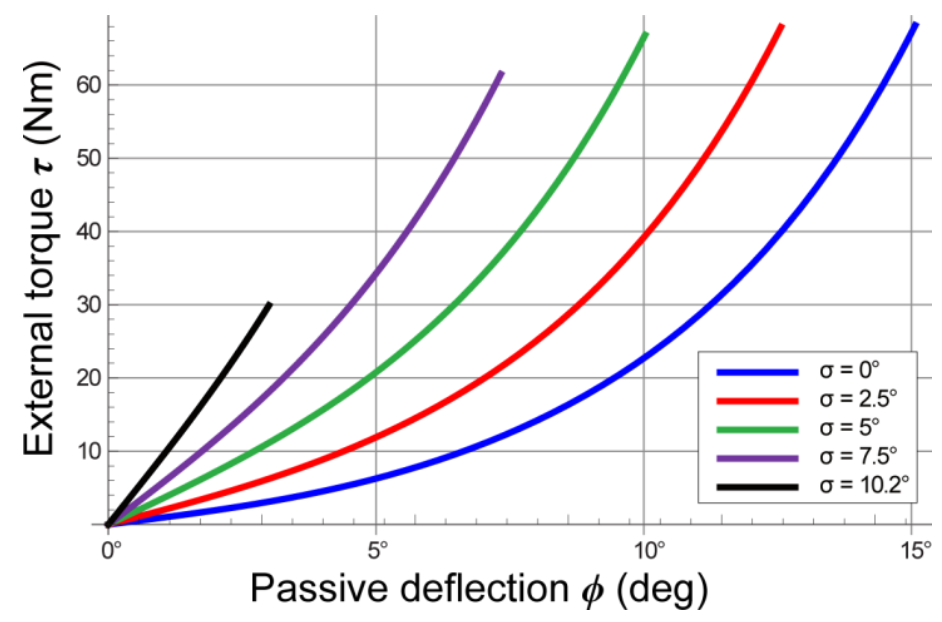

Fig. 20.4 The elastic torque characteristics of the DLR FSJ is parameterized by stiffness setup $\sigma$. The stiffness setup is set by the small motor. [4] 


\subsubsection{Flexible Antagonistic Spring Element (FAS)}

The FAS is motivated by the antagonistic arrangement of the human muscular system. Herein, two muscles act in opposing directions to the joint. To change the joint position, the muscles generate asymmetric forces. The stiffness can be varied by co-contraction of the muscles, which generate internal forces. In order to change the stiffness by co-contraction, the coupling between joint and muscles has to be a non-linear spring, for which reason the tendons are exponential spring elements [8].

In the FAS two motors of equal size are connected to the output by tendons. In the tendon routing, the tendons pass compliantly supported pulleys (see Fig. 20.5). The pulley located at the spring loaded lever (right pulley) rotates around the center of the guiding pulley (left pulley). The principle to move or stiffen the joint is the same as in the biological muscle arrangement [5]. It is depicted in Fig. 20.5a) in a low mechanical stiffness due to small $\alpha$ and in Fig. 20.5b) in a high stiffness due to a large $\alpha$.

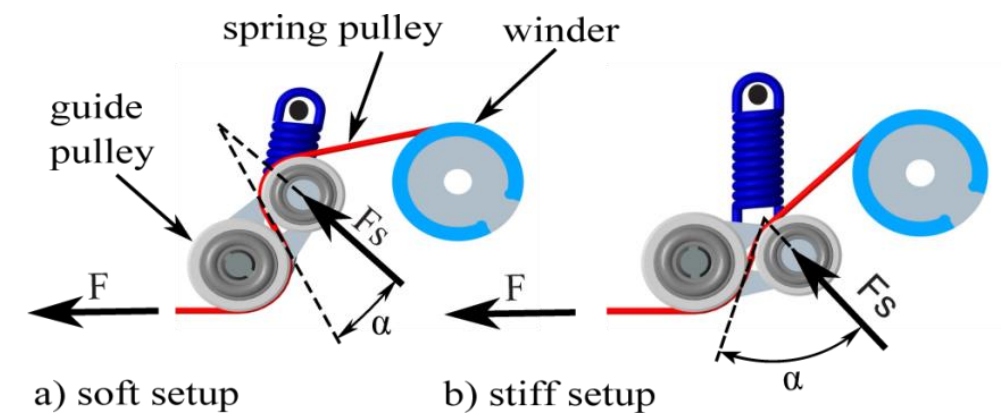

Fig. 20.5 Antagonistic drive compliance mechanism ("tan $\alpha$ mechanism") used for the FAS drive in a) a low mechanical stiffness, and b) a high stiffness. [5]

\subsubsection{Bidirectional Antagonism with Variable Stiffness (BAVS)}

The forearm rotation (pronation and supination) and both rotations of the wrist (radial and ulnar deviation; flexion and extension) are based on the principle of a Bidirectional Antagonism with Variable Stiffness (BAVS) [9], [10], [11]. A normal antagonistic mechanism has the drawback that only the power of one motor is available at the drive side of the joint. With the principle of BAVS we overcome this drawback by allowing both motors to push and pull and thus to assist each other in both directions. For this reason we also use the term helping antagonism (see Fig. 20.6). In the case of equally sized motors and gears, the output torque is twice the maximum individual motor torque. In the BAVS of the DLR Hand Arm System each motor is connected to a harmonic drive gear with the circular spline bedded in a ball bearing instead of fixing it to the base frame. In order to change 
the position of the VSA, both motors rotate in the same direction. In order to change the VSA stiffness, the motors move in the opposite direction, which causes a pretension in the non-linear springs, but keeps the output position constant. The non-linear spring characteristic is realized by a linear spring with a non-linear cam disc mechanism.

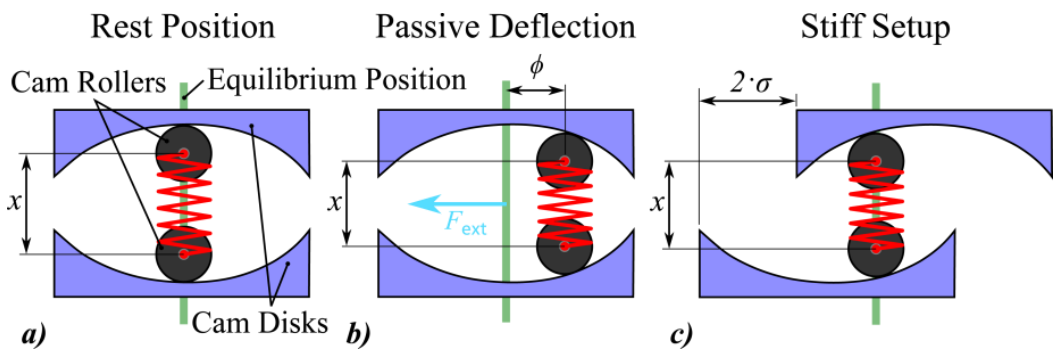

Fig. 20.6 BAVS drive: in a) the mechanism is in rest position, in b) the joint is deflected by an external torque, and in c) an equilibrium position in a stiff configuration is depicted (adapted from [2]).

The cam disks can be easily changed in order to test different stiffnessdeflection relations.

\subsection{Electronics and System Architecture}

Dealing with 52 actuators and 430 sensors of different types poses a challenge for electronics and systems architecture. Hence, major aspects as

- reliability and maintainability

- computation and communication bandwidth

- power density and integration level

have to be taken into account during the development and design process [2]. Therefore the system is designed modularly and hierarchically. Electronics as well as the computation and communication architecture follow this approach. Highly integrated electronic subcomponents which are independent and scalable guarantee the required flexibility, maintainability and reliability. This also is mirrored in a well-structured communication concept described in [2], [12], [13].

Fig. 20.7 shows the hierarchical topology of the DLR Hand Arm System. SpaceWire [14] is used as the communication backbone of the system. It is a standardized packet based bus with a bandwidth up to $1 \mathrm{Gbit} / \mathrm{s}$ and is deterministic for a given topology. Actuators and sensors are connected via the industry standard BiSS [15]. BiSS is a master/slave bidirectional serial bus with a typical data rate of $10 \mathrm{Mbit} / \mathrm{s}$ [2].The physical interfaces in turn are connected via their dedicated interfaces (I2C, SPI, PWM, etc.) to the sensor and actuator modules. Due to 
this hierarchical design the complexity of the system can be hidden from the application designer and on the other hand a good performance can be achieved with only a minimal execution overhead.

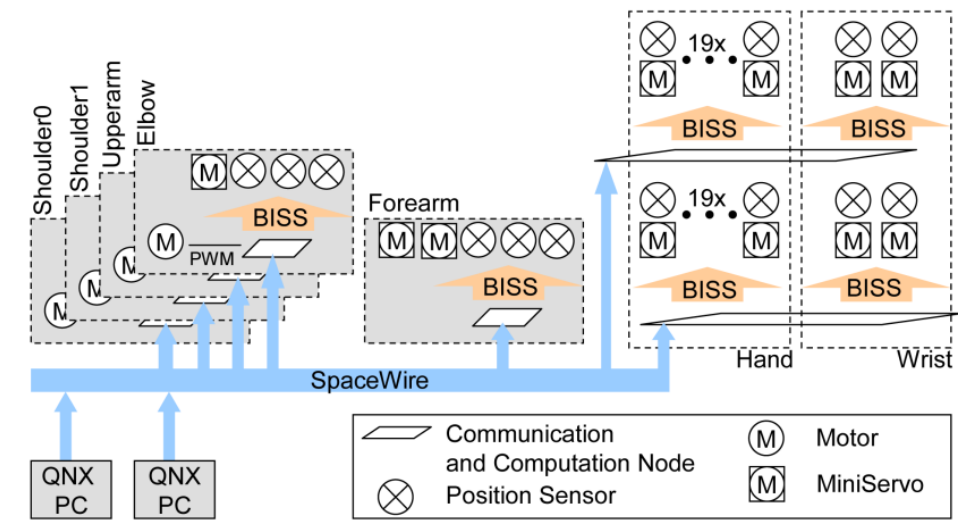

Fig. 20.7 DLR Hand Arm System topology. [2]

\subsection{Hand Design and Control}

The hand is the most exposed part of the robot, although it has the smallest force capabilities. The required features are large dexterity, manipulation capabilities, and robustness against unwanted collisions 259[16], [17]. As the human hand is highly under-actuated and uses several muscle/tendon synergies [18], which are not technically realizable, a plain copy of the human hand is not feasible. The hand design has to be based on an abstraction of the fundamental functionalities of the human hand. To reduce the number of drives, DoFs which have relatively low impact on grasping abilities should be omitted. These missing DoFs have to be compensated functionally by the kinematics design of the hand [19].

The kinematics of the hand (see Fig. 20.9) is closely adapted to the human hand on a functional basis [19]. It consists of 19 independent $\mathrm{DoFs}^{10}$ in order to reduce the number of necessary drives. Like human fingers, the index and middle finger have 4 DoFs. The 2 nd (PIP) and 3rd (DIP) finger joint ${ }^{11}$ of the ring and fifth finger are coupled to reduce the number of necessary actuators. The 5th DoF of the human thumb turned out to be of low relevance [20], [19] and have been omitted. To ensure proper opposition of the thumb and the 5th finger, an antagonistically driv-

\footnotetext{
10 Thumb 4 DoF, index finger 4 DoFs, middle finger 4 DoFs, ringfinger 3 DoFs, 5th finger 4 DoFs

11 starting from the fingers base
} 
en 4 bar mechanism was designed to mimic the motion of the 5th finger metacarpal bone ${ }^{12}$. The structure of the finger is designed as an endoskeleton with "bionic joints" [21]. The finger base (metacarpal) joint is a hyperbolically shaped saddle joint because the human condyloid joint type cannot be replicated technically ${ }^{13}$. The finger (interphalangeal) joints, on the other hand, are designed as hinge joints All joints allow dislocation without damage in case of overload ${ }^{14}$. In addition to robustness due to short-term energy storage, the use of antagonistic actuation (see Sec. 20.2) enables to cope with tendon slackening or overstretching, which is one of the major problems of nowadays tendon-driven hands having inevitably constant tendon length. In contrast, antagonistic actuation is able to compensate unaligned pulley axes, and other geometrical errors via the elastic elements of the drive train. Therefore no explicit tendon tensioner is needed [22].

The forearm is composed of 3 major parts: 1-DoF forearm rotation and 2-DoF wrist both with BAVS actuation and a 19-DoF hand with antagonistic actuation. The 38 motors and their corresponding nonlinear compliance mechanisms are tightly integrated into the forearm (see Fig. 20.8). The fingers are actuated via the ServoModules. A multiturn winder transfers the rotational gear motion to the tendons. The compliance mechanism is similar to the one described in [22]. One difference is that the winder also acts as the first pulley of the "tan $\alpha$ mechanism" (see Fig. 20.5). This reduces the number of required pulleys. Each compliance element is adapted to the different finger and joint characteristics. In order to facilitate maintenance, the finger actuators are placed on two almost identical halves. This configuration allows to replace ServoModules without dismounting the tendons. If a tendon replacement is required, the forearm can be opened to grant access to the compliance mechanisms as well as the winders.

\footnotetext{
12 the first bone of the finger located within the palm

13 in a meaningful way

14 the elongation of the tendons in case of dislocation is compensated by the elastic elements of the antagonistic drives
} 


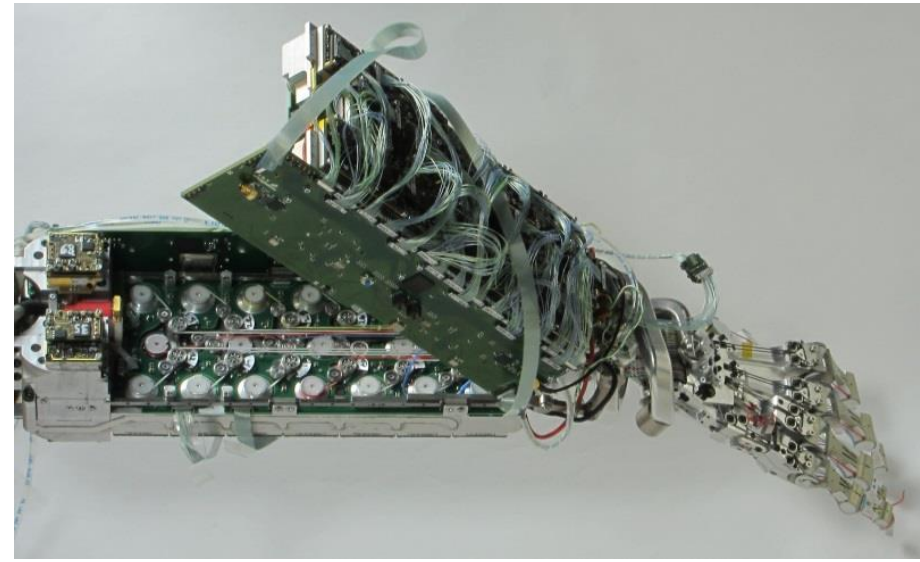

Fig. 20.8 Opened forearm with the ServoModules. The ServoModules are located on the outside of each half of the forearm. The tendons and the elastic elements are located in the middle layer between both halves.

The compact ServoModules are used for both the wrist and forearm rotation since the requirements are almost identical. The major difference is a higher required torque for the forearm rotation and wrist actuation than for the finger actuation. This requirement motivated the use of the newly developed BAVS actuation (see Sec. 20.2).

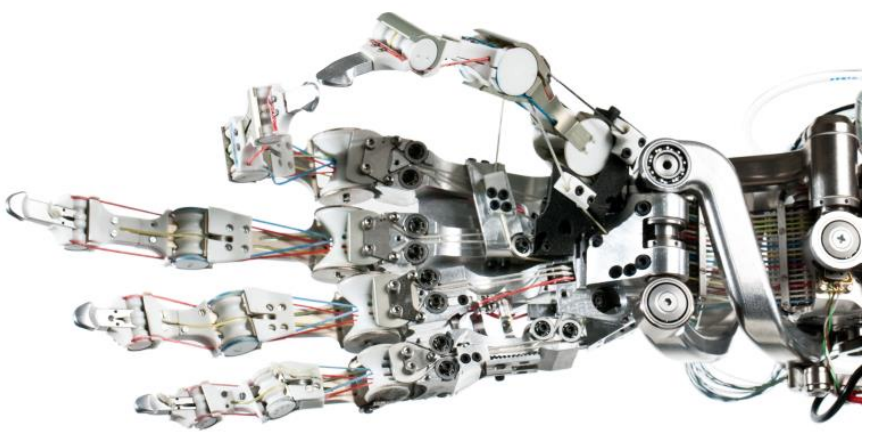

Fig. 20.9 Hand and wrist of the DLR Hand Arm System. 


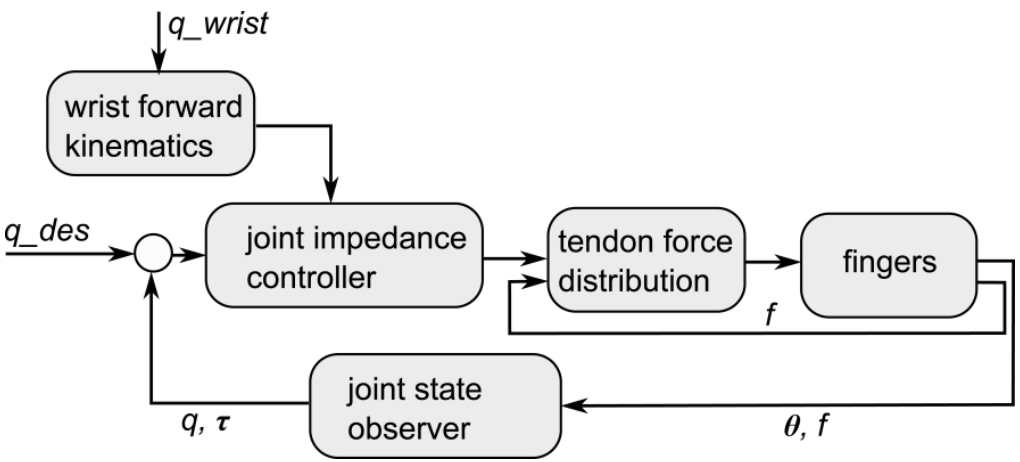

Fig. 20.10 Hand controller structure. $q$ and $\theta$ represent the joint and motor positions. $f$ and $\tau$ represent the tendon forces and the joint torques.

The hand controller implements a joint level impedance behavior to facilitate interactions with the environment. The top level control scheme implemented on the system is depicted in Fig. 20.10. The joint state observer provides an estimate of the joint position and the joint torque based on the measured motor displacement and the FAS sensors. An inner loop ensures that the tendon pulling constraints are holding and that the maximal tendon forces are not exceeded. The joint impedance controller is using a back-stepping structure in order to deal with the flexibility of the joints. A kinematic model of the wrist is used to compensate for the tendon displacement due to wrist motion.

\subsection{Modeling Soft Robots}

A soft robot with lumped elasticities such as the DLR Hand Arm System can be imagined as a set of directly actuated rigid bodies with configuration $\theta \in \mathbb{R}^{m}$, connected to the indirectly actuated rigid bodies (with configuration vector $\theta \epsilon \mathbb{R}^{n}$ ) through visco-elastic forces [23]. The entire configuration space of the system is denoted by $x=(\theta, q), x \in \mathbb{R}^{(n+m)}$. A quite general abstraction of a compliant robot, which can be used for the generic design of controllers, is given by

$$
M(x) \ddot{x}+c(x, \dot{x})+\frac{\partial V(x)}{\partial x}+d(\dot{x})=\left[\begin{array}{c}
\tau_{m} \\
\tau_{\mathrm{ext}}
\end{array}\right]
$$

with $M(x)$ being the inertia matrix, $c(x, \dot{x})$ the Coriolis and centrifugal vector, $V$ the potential energy of the elastic element and of the gravity forces, $\tau_{m} \in \mathbb{R}^{m}$ the actuator generalized forces acting as control inputs and $\tau_{e x t} \epsilon \mathbb{R}^{n}$ the external torques acting on the robot as a disturbance. The most relevant property of this structure is its 
under-actuation, meaning that the system has less control inputs $(m)$ than its configuration space dimension $(n+m)$. However, in contrast to other purely inertially coupled under-actuated systems ${ }^{15}$, for the considered robots $V(x)$ is [24] positive definite, implying that a unique equilibrium point exists for each external torque with actuators in a fixed configuration $\theta=\theta_{0}$ and that the linearization of the system around an equilibrium point $\left\{x=x_{0} ; \dot{x}=0\right\}$ is controllable. Typically, $V(x)=$ $V_{G}(x)+V_{\tau}(x)$, i.e. the potential function is the sum of a gravity potential $V_{G}$ and an elastic potential $V_{\tau}$. The elastic potential function $V_{\tau}(x)$ is a convex function, increasing strongly enough to compensate for the destabilizing effects of $V_{G}$, such that $V(x)$ is convex as well. Furthermore, the system contains in general a dissipative friction force $d(\dot{x})$ with $\dot{x}^{T} d(\dot{x}) \leq 0$.

If there is no substantial inertial coupling between motors and links and under some further mild simplification assumptions, the model can be written in a form resembling more the classical flexible joint robots:

$$
\left[\begin{array}{cc}
M(q) & 0 \\
0 & B
\end{array}\right]\left[\begin{array}{c}
\ddot{q} \\
\ddot{\theta}
\end{array}\right]+\left[\begin{array}{c}
c(q, \dot{q}) \\
0
\end{array}\right]+\left[\begin{array}{c}
g(q) \\
0
\end{array}\right]+\left[\begin{array}{c}
\tau \\
-\tau
\end{array}\right]+d(\dot{x})=\left[\begin{array}{c}
\tau_{\mathrm{ext}} \\
\tau_{m}
\end{array}\right]
$$

\subsection{Cartesian Stiffness Control}

\subsubsection{Cartesian Impedance Control}

While the mechanical stiffness of VIA robots can be adjusted in a wide range, not any task impedance can be realized by shaping the intrinsic mechanical properties. This is due to intrinsic limitation in minimal and maximal mechanical stiffness as well due to the fact that so far, most robots do not have coupled stiffness elements which would require multi-joint actuators [25]. This is in contrast to biological systems, which have a much larger number of muscles and also contain a large number of multi-articular joints. However, one can design a stiffness controller on motor side, which acts in serial interconnection with the intrinsic mechanical stiffness. Following a central paradigm of VIA, the mechanical robot parameters need to be tuned such that the desired behavior is naturally achieved on a mechanism level to the largest extent possible.

The stiffness behavior is described by a constant stiffness matrix $K_{C}=\frac{\partial f}{\partial x} \epsilon$ $\mathbb{R}^{m \times m}$ as the relation between the Cartesian wrench $f$ and the Cartesian displacement $x$. The $\mathrm{n}$ passive and adjustable joint stiffness components provide the diag-

15 as for example multiple pendulums such as the Acrobot [27] 
onal $^{16}$ matrix $K_{J}=\frac{\partial \tau}{\partial q} \epsilon \mathbb{R}^{n \times n}$ with the joint torques $\tau$ and the joint positions $q$. The mapping from the Cartesian stiffness space to the joint stiffness space is given by $\tau: K_{J}=T\left(K_{C}\right)$.

This transformation can be written as

$$
\begin{aligned}
K_{J} & =\frac{\partial \tau}{\partial q}=\frac{\partial\left(J(q)^{T} K_{C} \Delta x\right)}{\partial q} \\
& =J(q)^{T} K_{C} J(q)-\frac{\partial J(q)^{T} K_{C} \Delta x}{\partial q} .
\end{aligned}
$$

$J(q)=\frac{\partial f(q)}{\partial q}$ is the manipulator Jacobian, where $f(q)$ is the forward kinematics mapping. $\Delta x=x_{d}-x$ is the Cartesian position error between the desired and the actual position. The first part of (3) reflects the stiffness around the equilibrium point. The second part of (3) is due to the change of the Jacobian, see [26].

The Cartesian stiffness a the equilibrium position $(\Delta \mathrm{x}=0)$, resulting from a specific joint stiffness can be obtained by solving the inverse problem of (3), $K_{\mathrm{C}}=\tau^{-1}\left(\mathrm{~K}_{\mathrm{J}}\right)^{17}$. Using compliance matrices $C_{.}=K_{\bullet}^{-1}$, it results from

$$
\left.C_{C}=J(q) C_{J} J(q)^{T}\right)
$$

that

$$
K_{C}=\left(J(q) K_{J}^{-1} J(q)^{T}\right)^{-1} .
$$

Active control adapts the stiffness in a wider range, and the elastic elements are capable of absorbing impacts and increase the energy efficiency. By combining the two concepts, one can benefit from the individual advantages. The serial interconnection of the active stiffness $K_{\text {active }}$ and the passive one $K_{\text {passive }}$ results in an overall stiffness $K_{\text {res }}$ (see Fig. 20.11):

$$
K_{\text {res }}^{-1}=K_{\text {active }}^{-1}+K_{\text {passive }}^{-1}
$$

To compute the active and passive stiffness components, a two-step optimization algorithm can be used [28]. It achieves first a passive stiffness as close as possible to the desired one and secondly designs the active stiffness to minimize the residual.

\footnotetext{
16 Decoupled joint compliance is assumed.

${ }^{17}$ Note that at the equilibrium position the second term in 3 vanishes
} 


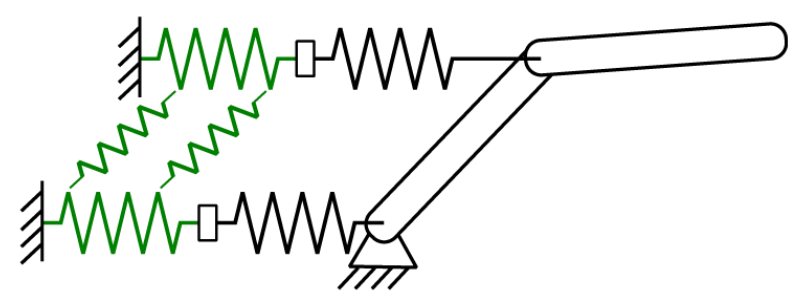

Fig. 20.11 Combination of active impedance controller with passive compliance to achieve a desired Cartesian stiffness.

\subsubsection{Independent Position and Stiffness Control}

The classical robot positioning task has some obvious particularities in the case of VIA systems. One does not only need to control the position of the link, but simultaneously has the chance to control the joint stiffness as well. This is possible due to the two independent actuators per joint. Additional control goals, especially important in the presence of disturbances, are the minimization of deflections and the suppression of vibrations.

The solutions to achieve independent control of link motion and stiffness can be classified in two categories.

- One class of controllers exploits the knowledge of system models. In [29] and [30] feedback linearization approaches are used to transform the robot dynamics into an equivalent model of simpler form. A decoupled chain of integrators can be achieved, as long as system inversion is possible. The simple structure of the equivalent dynamics allows for simultaneous decoupling and accurate tracking of motion and stiffness reference profiles. However, the abstraction of the robot dynamics hinders the implementation of performance criteria.

Another model based approach aims to achieve a reduced order model [31]. Therefore, separate dynamics are identified in the robot system, namely the arm, the positioning actuators, and the stiffness actuators. The independence of these dynamics is shown by a singular perturbation analysis. A cascaded control structure is based upon this analysis.

The abstraction that this class of controllers provides, allows for theoretical simplicity and design flexibility. On the other side, robot performance and robustness in the presence of model uncertainty are not guaranteed. High model accuracy and high derivatives of states are often required. Furthermore, to conform with the idea of embodiment additional effort is necessary, as the controllers often enforce a very different robot behavior than the natural one.

- The second class of controllers are energy shaping based controllers.

One of the first controllers in this category was presented by [32]. The control- 
ler acts on motor position and uses a transformation to independently control the joint position and stiffness. The controller is validated on a 1 DoF VSA joint.

An extension and generalization has been presented in [33]. The control design formulation is valid for a quite general form of underactuated Euler-Lagrange systems including variable impedance robots. Herein, the controller action can be interpreted as shaping of the potential and kinetic robot energy ensuring system passivity.

A general task is described as to control $\mathrm{k}$ independent output variables given by $q=h(x)$ to desired constant values $q_{d} \in R^{k} . \epsilon R^{n}$ is the vector of generalized coordinates, where $n=2 k$ is the usual case for VSA robots. Given the structure of VSA robots (2), a new variable $\bar{q}$ can be found. This is a collocated (directly actuated) variable, which is statically equivalent to the noncollocated (indirectly actuated) $q$. Using this collocated variable for a passive feedback ensures stability and can be interpreted as shaping the potential energy of the system.

The variable $\bar{q}$ is achieved by solving the static solution of the link side equation

$$
u=g(\bar{q})-J_{\bar{q}}^{T}(\theta) K_{p}\left(\bar{q}-q_{d}\right)-K_{d} \dot{\theta}
$$

for $q$. Except for very simple cases, this equation has to be solved numerically. Due to the convex nature, this is a fast and numerically robust task in practice. Consequently, the controller

$$
\tau+g(q)=0
$$

is stabilizing the desired position $q_{d} \cdot g(\bar{q})$ is a feed forward term, compensating for the gravity. The use of $\bar{q}$ enables arbitrarily low controller gains even for large displacements from the equilibrium. $J_{\bar{q}}$ is a Jacobian mapping the collocated variable on the statically equivalent $\bar{q}$. Global asymptotic stability based on La Salle's invariance theorem can be shown.

The approach can be extended in order to include also feedback of torque and torque derivative, with the effect of reducing the apparent actuator inertia and friction. This allows improving the transient performance while remaining within the passivity framework.

The energy shaping approach provides excellent performance in the static case and for well damped systems. However, some joints show low intrinsic damping to enable joint torque estimation and energy efficiency. In this case it is desirable to add additional damping via control or to include a physical variable damping element. Several damping control structures can be considered. A simple gain scheduling approach for a one DoF system has been presented in [34]. Local linear sub-problems are identified on which a LQR state feedback controller is designed. The nonlinearity of the robot dynamics requires adapting the controller poles dependent on the system state, which is especially hard for the multi joint robots. 
A physically motivated state feedback control approach for multi-DoF VSA robots has been presented in [6] using an eigenvalue based modal decomposition.

\subsection{Optimal Control}

Humans are capable of highly dynamic motions such as throwing or kicking. A major feature that presumably enables them to perform such tasks is their ability to optimally store and release elastic energy in the compliant elements of the musculoskeletal system, in combination with inertial energy transfer between the rigid parts of the body. Based on this feature, using elastic elements in robot joints, and finding control strategies which exploit this elasticity optimally has recently drawn significant attention.

The question of how to make use of the (adjustable) potential energy stored in elastic elements of a robotic system in the best possible way is not a trivial one, especially when considering the increased complexity of robotic systems with additional elasticity. A powerful mathematical tool for dealing with this question is Optimal Control (OC) Theory [35], which provides conditions for the control to minimize a given cost functional. Generally, a cost functional can be written as a sum of two terms

$$
J(u)=\vartheta\left(x\left(t_{f}\right), t_{f}\right)+\int_{t_{0}}^{t_{f}} L(x(t), u(t), t) \mathrm{d} t,
$$

where the first term, the terminal cost, considers the final state of the state and the terminal time, whereas the second term, the integral cost, takes the whole trajectory into account. By choosing an appropriate cost functional, the optimal cost can then be used as an indicator for the performance one can gain from elastic joints. Unfortunately, the conditions which OC theory provides, cannot always directly be formulated as control laws, especially when one wants to analyse a complex robotic system with multiple degrees of freedom and various nonlinearities. Nevertheless, under some simplifying assumptions, it is in some cases also possible to find analytical solutions, which are useful in understanding the best control strategies for these novel devices. Consequently, two main approaches are being followed for the analysis of these systems.

The first one is to work on simplified models such that analytical results can be obtained ([36][37][38][39][40][41][42][43][44][45]). These results are useful to reveal the relation between the system's parameters such as eigenfrequency, damping ratio and the maximum attainable performance regarding for instance the system's peak velocity during a throwing motion. The second approach is the use of powerful numerical tools such as pseudospectral methods [46], which have been successfully applied to various robotic systems including the DLR Hand Arm System ([37], [2], [48], [49]). 
Even though one can compute the optimal control strategy for complicated systems following the second approach, this computation may require a significant amount of time depending on the complexity of the used model. Consequently, for applications where the optimal control strategy is needed in real-time, these methods need to be further developed. In [50], the problem of generating optimal motions in real-time was addressed by encoding trajectories via Dynamic Movement Primitives (DMPs). The optimal trajectories learned via DMPs have the advantage of being reconstructable in real-time with high precision. Furthermore, the extrapolation to other tasks show near-optimal behavior, which has been illustrated in [50] by realizing a ball throwing motion with the DLR Hand Arm System, when the goal position is varying.

Comparing the existing results regarding the optimal control of elastic joints in literature, a lack of existing analytical results especially for n-DoF systems is apparent. Consequently, filling this gap and combining the gained insight for more efficient numerical methods and learning algorithms is an ongoing research.

\subsection{Collision Detection and Reaction}

As mentioned previously, intrinsic joint elasticity improves impact robustness In addition, the potential energy storage and release capabilities in the joints allow to outperform rigid manipulators by means of achievable peak link velocity. While high link velocities are desirable in terms of performance, they may also increase the robot's level of dangerousness and the risk of self-damage during collisions. In other words we have to consider both, threats for the environment and the robot. Threats for the environment are mainly caused by contact forces with the robot, whereas threats for the robot are dominated by the torque in the drive-train of the robot actuators. Thus, the problem of ensuring safety has to be treated differentiated. In physical human-robot interaction (pHRI) it is therefore important to detect contact with the environment and take appropriate countermeasures by control in case of unwanted collisions.

For detecting and isolating collisions with the DLR Hand Arm System, we use the generalized link momentum observer proposed in [51]. As this observer requires knowledge of the elastic joint torque, we use an estimate obtained by model identification. An alternative scheme described in [30] observes both the motor and link momentum and therefore does require identification of the joint torque. However, this scheme showed poor performance in practice because it is sensitive to unmodeled motor friction. Having detected collisions with the environment, collision reaction schemes can be activated to ensure the physical integrity of human and robot. 


\subsubsection{Reactions}

The most intuitive collision reaction is to stop the robot. For rigid robots or robots with high joint stiffness such as the LWR-III, stopping can simply be achieved by engaging the brakes or commanding the current position to the position controller. However, when halting the motors of VSA robots the links can still oscillate significantly due to the intrinsic joint elasticity. This can result in large link side velocities and even constraints such as maximum elastic deflection may be violated. The decrease of link velocity then depends on the damping of the joints, which is typically undesired for VSA robots and therefore very low.

Braking of elastic joint robots can be achieved by introducing active damping by control. For this, several schemes have been proposed recently. The state feedback controller described in [6] bases on a eigenmode decoupling approach and selects feedback gains such that dynamics and control behave like damped second order systems. A second decoupling based method described in [52] aims at braking an elastic robot as fast as possible, in other words, introducing as much damping as possible. The controller makes use of optimal control theory to brake each decoupled SISO mass-spring system in minimum time. It is noteworthy, that this optimal control problem can be solved in real-time. Both previously mentioned methods require a dynamic model of the robot. A model-free approach was presented in [7] were the energy storage and release process of every joint is exploited to form a passive damping controller.

An overload in the drive-train of a VSA robot typically results in the situation that the spring deflection limit is approached, e.g. by an external impact. In this case the mechanics or spring is likely damaged. In order to avoid this, an extremely large spring could be utilized, which would be of no use during the normal operations and would increase weight and bulkiness. An alternative is to actively move the VSA position in the direction of the threatening torque, so that the spring is discharged. This for example can be initiated at a load level above a given remaining potential energy capacity, or a remaining passive deflection angle (see red dashed line in Fig. 20.12), [53].

Another possible reaction to avoid spring overload takes advantage of the capability of a VSA to change its stiffness. It uses the same activation criteria as the previous reaction. Depending on the construction and the implementation principle, a VSA features different spring energy capacity or maximum deflection angle at different stiffness presents. If the VSA is set due to application demands to a less advantageous stiffness preset concerning the robustness issue, this can be mitigated by the reaction strategy. As long as the threat of overload is present the VSA changes the stiffness preset to a safer state and returns to the normal state when the critical situation is over. Spring preload type and antagonistic VSAs have a higher energy capacity at lower stiffness presets, because the energy used to preload the spring(s) for a stiffer setup cannot be used for passive deflection (light grey area in Fig. 20.12). In this case the motors release the preload during the reaction so that they do not have to supply additional power and at the same 
time increase the actuator energy capacity. An additional benefit is that also the maximum passive deflection angle increases with a softer stiffness preset.

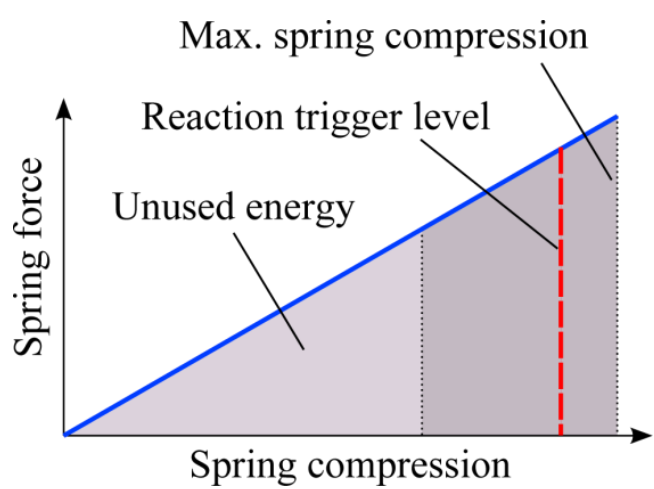

Fig. 20.12 Spring force vs. spring compression with the spring energy (grey area below the blue line) and the reaction trigger level (red line).

\subsubsection{Reflexes}

A novel approach to increase safety and security of VSA systems is to take the human mimetic approach one step further: The actuator unit consisting of motor and spring can be controlled analogously to the human muscle and tendon, which are protected by reflex actions. Those reflex actions can be interpreted on a control level to protect the mechanical VSA system. But what are those protective reflexes and what do they regulate? Among them is the stretch reflex, commonly known from the tendon jerk experiment: A tap on the tendon of the quadriceps passively stretches the muscle and special receptors, the so called muscle spindles, lead to a contraction of the same muscle. Moreover there is the more complex nociceptive withdrawal reflex that leads to a withdrawal movement elicited by a painful stimulus. The number of activated muscles is scaled with the intensity of the stimulus and might lead to the retreat of only one limb, e.g. after tapping on a needle, up to a reaction of the whole body jumping away from the source of stimulus. A third reflex is the autogenic inhibition that leads to a relaxation of the muscle threatened by overload: If a force is much too strong for a muscle, golgi tendon organs induce its inhibition to protect it from harmful tear.

Those reflexes are very different, but our assumption is that they follow common principles, which are namely: hierarchy, since they operate concurrently in a predefined order, where the central nervous system (CNS) is at the highest level and can suppress any reflex activity by conscious thought; operation on joint level and not necessarily a reaction in Cartesian space; reflex reversal, which means that the same stimulus can lead to different reactions, depending on the situation; irradiation, because the stronger a stimulus the more muscles are activated and the re- 
action is spread (irradiated) over the joints; the preservation of a status quo, which is e.g. stability or sanity. The reflexes, interpreted on a control level, are a combination of PD control (stretch reflex), force/torque control (autogenic inhibition) and a fast trajectory (nociceptive withdrawal reflex). We propose an activation strategy of the reflexes based on two inputs: The measured deflection of the spring mechanism, directly correlating to the torque, since the stiffness of the spring is known, and the force input from an artificial skin. The activation is complemented with a switching strategy of the control mode that preserves the stability of the system in action. Thus, the system is PD controlled and moves away from a source of stimulus to the artificial skin that is arranged in so called reflex responsive fields, eliciting a movement of the proximal joint away from the source of stimulus (see Fig. 20.13). Two of the use cases are: (A) An evasive trajectory of the motor after an impact on the artificial skin to a new set-point, symbolized by a red dot; (B) A switch to torque control mode after over-lengthening of the spring to reduce the stored elastic energy. The control modes can be used conjointly and the reaction can be spread over multiple proximal joints. After each reflexive reaction a trajectory back to original set-point, symbolized by a green dot, is computed. The stronger the stimulus, the more joints are activated to support the movement. If overload of the spring is detected, the system switches into torque control, hence reducing the energy storage in the spring, and switches back to PD control, after necessary energy and stability conditions are satisfied. All control action takes place on joint level, can be spread over multiple proximal joints as well as suppressed by raising the activation-thresholds. It only steps in when necessary and thus enhances existing control schemes by providing additional safety and security.

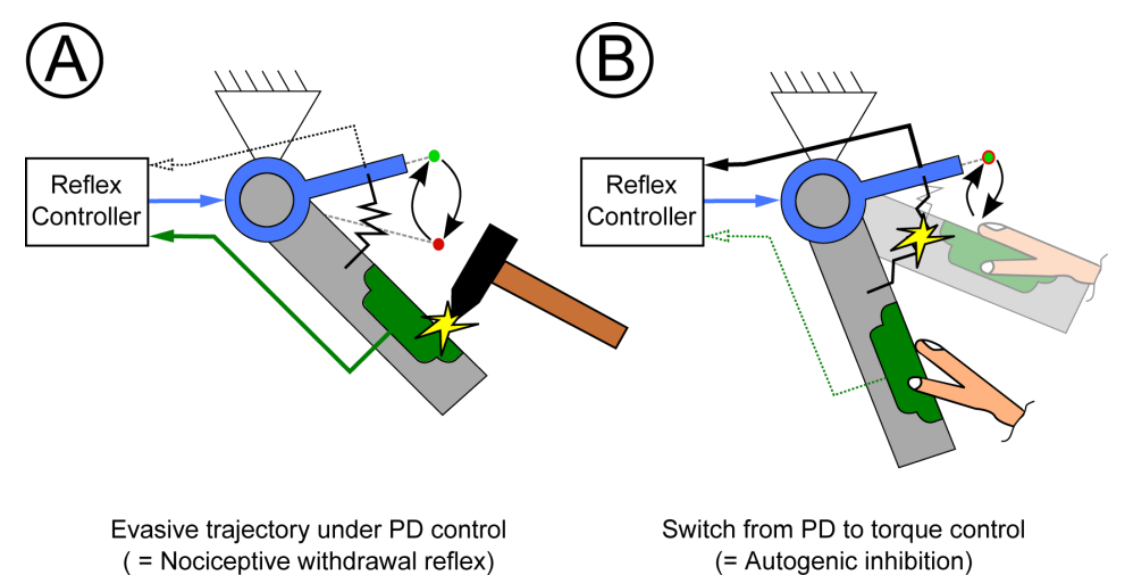

Fig. 20.3 Reflex use cases for a VSA joint, where the motor is depicted in blue, the link in grey, a passive compliance as black spring and the artificial skin in green. 


\subsection{Cyclic Motion Control}

Key properties of compliant actuators in robotic arms are the capability to improve performance and energetic efficiency. Especially in case of cyclic motion tasks, the capability to buffer and release elastic energy may reduce the size and weight of required actuators and save a substantial amount of energy. These properties are even of increased importance for legged robots, which need to wirelessly walk, jump, or run over rough and uneven terrain. The step from rigid towards elastic actuation introduces natural oscillation dynamics into the plant. In our recent works [54], [55], [56], [57], [58], [58], novel concepts to exploit these natural dynamics have been derived.

Considered limit cycle control methods range from classical principles based on the well-known Van der Pol oscillator to novel concepts following the idea of resonance and natural dynamics excitation. The former method is adapted from rigid actuator control, where the generalized force of the second-order dynamics can be considered as control input. An implementation for compliant actuators is proposed in [54]. The latter method is derived from human motor control [56], [58]. This method directly applies to continuous second-order systems ${ }^{18}$ of the form

$$
m \ddot{q}+d(q, \dot{q})=-\left.\frac{\partial U(\phi)}{\partial \phi}\right|_{\phi=q-\theta},
$$

with positive plant parameters ${ }^{19}$ representing a large class of compliantly actuated robotic joints. The limit cycle controller is simply the switching law ${ }^{20}$.

$$
\theta\left(q, \theta^{-}\right)= \begin{cases}-\operatorname{sign}\left(q-\theta^{-}\right) \hat{\theta} & \text { if }\left|q-\theta^{-}\right|>\epsilon \\ 0 & \text { otherwise }\end{cases}
$$

For the maximally efficient controller parameters $\hat{\theta} \leq 2 \epsilon$, this controller generates a globally asymptotically stable limit cycle without any model knowledge, only based upon measurements of the spring deflection [58].

The extension of the above methods from the single to the multiple joint case under the concept of natural dynamics exploitation is derived from the notion of intrinsic mechanical oscillation modes [58]. Exploiting the idea of two dimensional invariant submanifolds, $2 n^{\text {th }}$-order dynamics of multiple joint robots collapses to second-order dynamics of a single joint. This dimensionality reduction can be achieved using one of the following approaches:

\footnotetext{
18 The system has continuous states $q, \dot{q} \in \mathbb{R}$

${ }^{19}$ The inertia $\mathrm{m}>0$, the generalized damping force is positive semi-definite in a sense that $\mathrm{d}(\mathrm{q}, \mathrm{q}$ ) $q \geq 0$ and the elastic potential $U(\phi)$ is positive definite w.r.t. the spring deflection $\phi$.

${ }^{20}$ The finite dynamics is piecewise continuous from the left, i.e. $\theta^{-}$represents the state of $\theta$ before the switching.
} 
- Considering a locally valid linearization of the nonlinear plant dynamics, linear eigenmodes can be identified. The valid range of these modes can be extended to hold globally by feedback control [54].

- On the basis of the switching control, a directed excitation along an intrinsic mechanical oscillation mode can be achieved by adaptive control [55].

- A method to generate a limit cycle along a predefined oscillation mode has been derived from classical impedance and null-space control [36].

The above methods can be nearly arbitrarily combined to achieve a multiple degree of freedom limit cycle control. One can adapt to one of the following requirements: task adaptability, performance, or efficiency.

\subsection{Conclusion}

We highlighted in this chapter that intrinsic flexibility in robotic systems is of crucial importance to obtain human level power density and to interact safely with humans and unknown environments. Using classical electro-mechanical actuation in combination with nonlinear elastic joint mechanisms allows realizing high performance actuators, with high motion repeatability and high efficiency. A large amount of control literature on flexible joint robots can be therefore accessed and adapted to the nonlinear, adjustable stiffness case. In this chapter we addressed on the basis of the DLR Hand Arm System all major aspects related to the mechatronic design and control of VSA robots. As a research prototype, the DLR Hand Arm System fully validates the feasibility of the VSA concepts. A major drawback remains the relatively high complexity of the nonlinear elastic joint. New technological approaches to implement the variable elastic element and integrated electronics solutions would therefore support the evolution of VSA systems towards commercial applications.

\subsection{References}

[1] Vanderborght B, Albu-Schäffer A, Bicchi A, Burdet E, Caldwell D, Carloni R, Catalano M, Eiberger O, Friedl W, Ganesh G, Garabini M, Grebenstein M, Grioli G, Haddadin S, Höppner H, Jafari A, Laffranchi M, Lefeber D, Petit F, Stramigioli S, Tsagarakis N, Damme MV, Ham RV, Visser L, Wolf S (2013) Variable impedance actuators: A review. Robotics and Autonomous Systems 61(12):1601-1614, DOI http://dx.doi.org/10.1016/j.robot.2013.06.009

[2] Grebenstein M, Albu-Schäffer A, Bahls T, Chalon M, Eiberger O, Friedl W, Gruber R, Haddadin S, Hagn U, Haslinger R, Höppner H, Jörg S, Nickl M, Nothhelfer A, Petit F, Reill J, Seitz N, Wimböck T, Wolf S, Wüsthoff T, Hirzinger G (2011) The DLR Hand 
Arm System. In: IEEE International Conference on Robotics and Automation (ICRA), pp 3175-3182, DOI 10.1109/ ICRA.2011.5980371

[3] Wolf S, Hirzinger G (2008) A new variable stiffness design: Matching requirements of the next robot generation. In: Robotics and Automation, 2008. ICRA 2008. IEEE International Conference on, Pasadena, CA, USA, pp 1741-1746, DOI 10.1109/ROBOT.2008.4543452

[4] Wolf S, Eiberger O, Hirzinger G (2011) The DLR FSJ: Energy based design of a variable stiffness joint. In: Robotics and Automation (ICRA), 2011 IEEE International Conference on, pp 5082-5089, DOI 10.1109/ICRA.2011.5980303

[5] Friedl W, Chalon M, Reinecke J, Grebenstein M (2011) FAS A flexible antagonistic spring element for a high performance over. In: Intelligent Robots and Systems (IROS), 2011 IEEE/RSJ International Conference on, pp 1366-1372, DOI 10.1109/IROS.2011.6094569

[6] Petit F, Albu-Schäffer A (2011) State feedback damping control for a multi dof variable stiffness robot arm. In: IEEE Int. Conf. on Robotics and Automation (ICRA), IEEE, pp $5561-5567$

[7] Petit F, Ott C, Albu-Schäffer A (2014) A model-free approach to vibration suppression for intrinsically elastic robots. In: IEEE Int. Conf. on Robotics and Automation (ICRA), IEEE

[8] Cui L, Maas H, Perreault EJ, Sandercock TG (2009) In situ estimation of tendon material properties: Differences between muscles of the feline hindlimb. Journal of Biomechanics 42(6):679 - 685, DOI http://dx.doi.org/10.1016/j. jbiomech.2009.01.022

[9] Friedl W, Höppner H, Petit F, Hirzinger G (2011) Wrist and forearm rotation of the DLR Hand Arm System: Mechanical design, shape analysis and experimental validation. In: Intelligent Robots and Systems (IROS), 2011 IEEE/RSJ International Conference on, pp 1836-1842, DOI 10.1109/IROS. 2011.6094616

[10] Petit F, Chalon M, Friedl W, Grebenstein M, Albu-Schäffer A, Hirzinger G (2010) Bidirectional antagonistic variable stiffness actuation: Analysis, design amp; implementation. In: Robotics and Automation (ICRA), 2010 IEEE International Conference, pp 4189 4196, DOI 10.1109/ROBOT.2010.5509267

[11] Petit F, Friedl W, Höppner H, Grebenstein M (2014) Analysis and synthesis of the bidirectional antagonistic variable stiffness mechanism. Mechatronics, IEEE/ASME Transactions on PP(99):1-12, DOI 10.1109/TMECH.2014. 2321428

[12] Jörg S, Nickl M, Nothhelfer A, Bahls T, Hirzinger G (2011) The computing and communication architecture of the DLR hand arm system. In: Proceedings IEEE/RSJ International Conference on Intelligent Robots and Systems, pp 1055-1062

[13] Nickl M, Jörg S, Bahls T, Nothhelfer A, Strasser S (2011) Spacewire, a backbone for humanoid robotic systems. In: Proceedings of the 4th International SpaceWire Conference, pp 356-359

[14] European Cooperation for Space Standardization (ECSS) (2003) ECSS E-50-12A SpaceWire - Links, nodes routers and networks. http://spacewire.eas.int

[15] IC Haus (2007) BiSS C Interface Protocol (C-Mode). http://www.ichaus.com, c1 edn

[16] Grebenstein M (2014) Approaching Human Performance: The Functionality-Driven Awiwi Robot Hand (Springer Tracts in Advanced Robotics). Springer, Berlin; Heidelberg; New York

[17] Grebenstein M, Chalon M, Friedl W, Haddadin S, Wimböck T, Hirzinger G, Siegwart R (2012) The hand of the DLR Hand Arm System: Designed for interaction. IJRR 31(13):1531-1555

[18] Gray H (1999) Anatomy, descriptive and surgical. Courage Books, Philadelphia

[19] Grebenstein M, Chalon M, Hirzinger G, Siegwart R (2010) A method for hand kinematics designers; 7 billion perfect hands. International Conference on Advances in Bioscience and Bioengineering 
[20] Chalon M, Grebenstein M, Wimböck T, Hirzinger G (2010) The thumb: Guidelines for a robotic design. Intelligent Robots and Systems, (2010) IEEE/RSJ International Conference on, pp 2153-2858

[21] Grebenstein M, Chalon M, Hirzinger G, Siegwart R (2010) Antagonistically driven finger design for the anthropomorphic DLR Hand Arm System. Humanoids Robots, IEEE/RAS International Conference, pp 609-616

[22] Grebenstein M, van der Smagt P (2008) Antagonism for a highly anthropomorphic handarm system. Advanced Robotics 1(22):39-55, DOI 10.1163/156855308X291836

[23] Albu-Schäffer A, Wolf S, Eiberger O, Haddadin S, Petit F, Chalon M (2010) Dynamic modelling and control of variable stiffness actuators. In: Robotics and Automation (ICRA), 2010 IEEE International Conference on, pp $2155-2162$, DOI 10.1109/ROBOT.2010.5509850

[24] Jafari A, Tsagarakis N, Caldwell D (2013) A novel intrinsically energy efficient development of a novel actuator with adjustable stiffness (awas). IEEE Transactions on Mechatronics $18(1)$

[25] Albu-Schäffer A, Fischer M, Schreiber G, Schoeppe F, Hirzinger G (2004) Soft robotics: What cartesian stiffness can we obtain with passively compliant, uncoupled joints? In: Proc. of the IEEE/RSJ Int. Conf. on Intelligent Robots and Systems, pp 3295-3301

[26] Hogan N (1990) Mechanical impedance of single- and multi-articular systems. In: Winters J,Woo SY (eds) Multiple Muscle Systems, Springer New York, pp 149-164

[27] Fantoni I, Lozano R, Spong MW (2000) Energy based control of the pendubot. IEEE Trans on Automatic Control 45(4):725-729

[28] Petit F, Albu-Schäffer A (2011) Cartesian impedance control for a variable stiffness robot arm. In: Proc. of the IEEE/RSJ International Conference on Intelligent Robots and Systems, pp 4180-4186

[29] Palli G, Melchiorri C, Luca AD (2008) On the feedback linearization of robots with variable joint stiffness. In: Proc. IEEE Int. Conf. on Robotics and Automation, pp 1753 1759

[30] De Luca A, Flacco F, Bicchi A, Schiavi R (2009) Nonlinear decoupled motion-stiffness control and collision detection/reaction for the vsa-ii variable stiffness device. In: IEEE/RSJ Int. Conf. on Intelligent Robots and Systems (IROS2009), IEEE, pp 54875494

[31] Palli G, Melchiorri C (2011) Output-based control of robots with variable stiffness actuation. Journal of Robotics

[32] Tonietti G, Schiavi R, Bicchi A (2005) Design and control of a variable stiffness actuator for safe and fast physical human/robot interaction. In: Proc. IEEE Int. Conf. on Robotics and Automation, pp 528-533

[33] Albu-Schäffer A, Ott C, Petit F (2012) Energy shaping control for a class of underactuated euler-lagrange systems. In: IFAC Symposium on Robot Control

[34] Sardellitti I, Medrano-Cerda G, Tsagarakis NG, Jafari A, Caldwell DG (2012) A position and stiffness control strategy for variable stiffness actuators. In: Proc. IEEE Int. Conf. on Robotics and Automation

[35] Pontryagin L (1987) Mathematical Theory of Optimal Processes. Classics of Soviet Mathematics, Taylor \& Francis

[36] Garabini M, Passaglia A, Belo FAW, Salaris P, Bicchi A (2011) Optimality principles in variable stiffness control: the VSA hammer. 2011 IEEE/RSJ International Conference on Intelligent Robots and Systems (IROS2011), San Francisco, USA pp 3770 - 3775

[37] Garabini M, Passaglia A, Belo F, Salaris P, Bicchi A (2012) Optimality principles in stiffness control: The VSA kick. In: Robotics and Automation (ICRA), 2012 IEEE International Conference on, pp 3341-3346

[38] Haddadin S, Weis M, Albu-Schäffer A, Wolf S (2011) Optimal control for maximizing link velocity of robotic variable stiffness joints. In: Proceedings IFAC 2011, World Congress pp 3175-3182 
[39] Haddadin S, Krieger K, Mansfeld N, Albu-Schäffer A (2012) On impact decoupling properties of elastic robots and time optimal velocity maximization on joint level. In: Intelligent Robots and Systems (IROS), 2012 IEEE/RSJ International Conference, pp 5089-5096, DOI 10.1109/IROS.2012.6385913

[40] Haddadin S, Özparpucu M, Albu-Schäffer A (2012) Optimal control for maximizing potential energy in a variable stiffness joint. In: Decision and Control (CDC), 2012 IEEE 51st Annual Conference, pp 1199-1206

[41] Incaini R, Sestini L, Garabini M, Catalano MG, Grioli G, Bicchi A (2013) Optimal control and design guidelines for soft jumping robots: Series elastic actuation and parallel elastic actuation in comparison. In: IEEE International Conference on Robotics and $\mathrm{Au}-$ tomation (ICRA2013), pp 2477 - 2484

[42] Özparpucu MC, Albu-Schäffer A (2014, Accepted) Optimal control strategies for maximizing the performance of variable stiiffness joints with nonlinear springs. In: Decision and Control (CDC), 2014 IEEE 53rd Annual Conference on

[43] Özparpucu MC, Haddadin S (2013) Optimal control for maximizing link velocity of visco-elastic joints. In: Intelligent Robots and Systems (IROS), 2013 IEEE/RSJ International Conference on, pp 3035-3042

[44] Özparpucu MC, Haddadin S (2014) Optimal control of elastic joints with variable damping. In: Control Conference (ECC), 2014 European, pp 2526-2533

[45] Özparpucu MC, Haddadin S, Albu-Schaffer A (2014, Accepted) Optimal control of variable stiffness actuators with nonlinear springs. In: Proceedings. IFAC 2014, World Congress

[46] Garg D, Patterson MA, Hager WW, Rao AV, Benson DA, Huntington GT (2010) A unified framework for the numerical solution of optimal control problems using pseudospectral methods. Automatica 46(11):1843-1851

[47] Braun D, Howard M, Vijayakumar S (2011) Exploiting variable stiffness in explosive movement tasks. In: Proceedings of Robotics: Science and Systems (RSS2011), Los Angeles, USA, pp 25-32

[48] Haddadin S, Huber F, Albu-Schäffer A (2012) Optimal control for exploiting the natural dynamics of variable stiffness robots. In: Robotics and Automation (ICRA), 2012 IEEE International Conference, pp 3347-3354, DOI 10.1109/ICRA.2012.6225190

[49] Mettin U, Shiriaev A (2011) Ball-pitching challenge with an underactuated two-link robot arm. Proceedings IFAC 2011, World Congress pp 1-6

[50] Weitschat R, Haddadin S, Huber F, Albu-Schäffer A (2013) Dynamic optimality in realtime: A learning framework for near-optimal robot motions. In: Intelligent Robots and Systems (IROS), 2013 IEEE/RSJ International Conference on, pp 5636-5643

[51] De Luca A, Albu-Schäffer A, Haddadin S, Hirzinger G (2006) Collision detection and safe reaction with the DLR-III lightweight manipulator arm. In: IEEE/RSJ Int. Conf. on Intelligent Robots and Systems (IROS2006), IEEE, pp 1623-1630

[52] Mansfeld N, Haddadin S (2014) Reaching desired states time-optimally from equilibrium and vice versa for visco-elastic joint robots with limited elastic deflection. In: IEEE/RSJ Int. Conf. on Intelligent Robots and Systems (IROS2014), IEEE

[53] Wolf S, Albu-Schäffer A (2013) Towards a robust variable stiffness actuator. In: Intelligent Robots and Systems (IROS), 2013 IEEE/RSJ International Conference on, IEEE/RSJ, Tokyo, Japan, pp 5410-5417

[54] Lakatos D, Garofalo G, Petit F, Ott C, Albu-Schäffer A (2013) Modal limit cycle control for variable stiffness actuated robots. In: Proc. IEEE Int. Conf. on Robotics and Automation, pp 4934-4941

[55] Lakatos D, Görner M, Petit F, Dietrich A, Albu-Schäffer A (2013) A modally adaptive control for multi-contact cyclic motions in compliantly actuated robotic systems. In: Proc. IEEE/RSJ Int. Conf. on Intelligent Robots and Systems, pp 5388-5395 
[56] Lakatos D, Petit F, Albu-Schäffer A (2013) Nonlinear oscillations for cyclic movements in variable impedance actuated robotic arms. In: Proc. IEEE Int. Conf. on Robotics and Automation, pp 508-515

[57] Lakatos D, Garofalo G, Dietrich A, Albu-Schäffer A (2014) Jumping control for compliantly actuated multilegged robots. In: Proc. IEEE Int. Conf. on Robotics and Automation

[58] Lakatos D, Petit F, Albu-Schäffer A (2014) Nonlinear oscillations for cyclic movements in human and robotic arms. IEEE Transactions on Robotics pp 865-879

[59] Lakatos D, Albu-Schäffer A (2014) Switching based limit cycle control for compliantly actuated second-order systems. Accepted for publication at the IFAC World Congress 